\title{
Pay for Performance: Aus Sicht von spectrum |K ein geeignetes Instrument für Qualitätsverbesserungen im Gesundheitssystem
}

\section{Ausgangsbetrachtung \\ $\nabla$}

Während heute in fast allen anderen Branchen erfolgsorientierte Vergütungskomponenten wie Boni, Umsatzbeteiligungen bzw. Konventionalstrafen als Malus üblich sind, haben sich derartige Vergütungsmodelle in unserem heutigen Gesundheitssystem kaum durchgesetzt. In einer Befragung $(n=182)$ von Ärzten zur Ausprägung von vereinbarten Vergütungssystemen in der Integrierten Versorgung (IGV) entfiel auf die Nennung von Bonus/Malus-Systemen nur knapp $2 \%$. An der Spitze der Nennungen lagen Fallpauschalen mit knapp 21 \% sowie die Einzelleistungsvergütung mit knapp $32 \%$ [2].

\section{Erfolgsabhängige Vergütung bei der künstlichen Befruchtung \\ $\checkmark$}

Im Jahr 2006 hat der BKK Landesverband Bayern mit dem Berufsverband Reproduktionsmedizin einen IGV-Vertrag mit erfolgsorientierter Vergütung zur künstlichen Befruchtung abgeschlossen. Bei diesem P4P-Modell erhalten die Ärzte eine Zusatzvergütung von ca. $1500,-€$, wenn eine Schwangerschaft eintritt. Der Erfolg definiert sich auch über möglichst wenige Zwillingsgeburten. Drillingsgeburten sind durch eine Beschränkung des Transfers auf höchstens 2 Embryonen komplett ausgeschlossen. Die erfolgsunabhängige Basisvergütung wurde im Gegenzug um ca. $30 \%$ abgesenkt [1]. Bei diesem Beispiel sind die Parameter zur Beurteilung von Erfolg und Misserfolg, nämlich schwanger vs. nicht schwanger, leicht erkenn- und überprüfbar. Dies ist jedoch die Ausnahme und nicht die Regel.

\section{Das P4P Model von spectrum |K beim Versichertencoaching \\ $\nabla$}

Für das telefonische Versichertencoaching hat spectrum|K eine Ausschreibung vorbereitet, mit dem Ziel, den Kassen Rahmenverträge mit geeigneten Dienstleistern anbieten zu können. spectrum|K hat dabei ein innovatives Vergütungsmodell mit einem P4P-Ansatz entwickelt. Das Vergütungsmodell besteht aus 4 Komponenten:

1. Einer Basisvergütung mit Mengenrabatten

2. Einer Risikobeteiligung als Anteil der Basisvergütung

3. Einer Return on Invest (RoI) bezogenen

Erfolgsbeteiligung

4. Einer RoI bezogenen Erfolgsgarantie
Unter der Basisvergütung (1.) wird ein aufwandsbezogener Festbetrag pro gewonnenem und pro betreutem Kandidaten verstanden. Darauf wird ein Mengenrabatt, differenziert nach der Anzahl betreuter Patienten gewährt. Als Risikobeteiligung oder Malus (2.) ist ein prozentualer Anteil der Basisvergütung vorgesehen, welcher sich aus der Anzahl erreichter bzw. nicht erreichter Servicepunkte errechnet. Die Servicepunkte resultieren aus aktivitätsbezogenen, qualitätsbezogenen und ökonomischen Evaluierungsparametern. Als Erfolgsbeteiligung oder Bonus (3.) sollen die Dienstleister am ökonomischen Erfolg beteiligt werden. Wenn ein Dienstleister ein über dem vertraglich festgelegten und garantierten RoI (4.) liegendes Ergebnis realisieren kann, wird er prozentual am darüber liegenden Erfolg beteiligt.

Die Entwicklung eines solchen Vergütungsmodels ist kompliziert, und die Parameter zur Beurteilung von Erfolg und Misserfolg sind in diesem Fall nur mit einer relativ aufwendigen Evaluation zu ermitteln. Vermutlich sind dies auch wesentliche Gründe dafür, dass sich P4P im heutigen Gesundheitssystem bisher wenig durchgesetzt hat. Aus Sicht von spectrum|K ist die qualitätsabhängige Vergütung jedoch ein wichtiger Ansatz zur Qualitätsverbesserung sowohl im kollektivvertraglichen wie im selektiv-vertraglichen Bereich.

Autorenerklärung: Der Autor erklärt, dass keine relevanten finanziellen Verbindungen in Bezug auf dieses Manuskript bestehen.

\footnotetext{
Literatur

1 Braun et al. Innovative Versorgungsformen im Gesundheitswesen - Konzepte und Praxisbeispiele erfolgreicher Finanzierung und Vergütung. Köln: Deutscher Ärzte-Verlag, 2009: 121-130.

2 Pitum-Weber S. Management von Gesundheitsnetzwerken. Dissertation 2008
}

\section{R. Leuschner}

Qualitätsmanagement

Schlüsselwörter

OP4P

Versichertencoaching

Bonus Malus

Erfolgsabhängige Vergütung

Keywords

pay for performance

payment incentives

quality performance assessment

Institut

spectrum|K GmbH, Berlin

Bibliografie

DOI $10.1055 / \mathrm{s}-0029-1242676$

Dtsch Med Wochenschr 2009;

134: S321 . (c) Georg Thieme

Verlag KG Stuttgart - New York . ISSN 0012-0472

Korrespondenz

Dr. Roland Leuschner spectrum |K GmbH Spittelmarkt 12

10117 Berlin

Tel. 030/212336-273

eMail roland.leuschner@ spectrumk.de 